\title{
Stage IB1 Cervical Cancer AJCC v6 and v7
}

National Cancer Institute

\section{Source}

National Cancer Institute. Stage IB1 Cervical Cancer A/CC v6 and v7. NCI Thesaurus.

Code C6317.

Stage IB1 includes: T1b1, N0, M0. T1b1: Clinically visible lesion $4.0 \mathrm{~cm}$ or less in greatest dimension. N0: No regional lymph node metastasis. M0: No distant metastasis. (AJCC 6th and 7th eds.) 